\title{
Desempenho de SACs cultivados com forrageiras, quanto à remoção de matéria orgânica de esgoto doméstico
}

\begin{abstract}
Dentre as alternativas para tratamento de efluentes, visando minimizar problemas decorrentes da falta de saneamento, vêm se destacando o uso de wetlands construídos, os quais, além de apresentar baixo custo de instalação, ser ecológico, possuem efeitos paisagísticos de fácil aceitação para quem adere a técnica. Assim, este trabalho teve como objetivo avaliar a eficiência de sistemas alagados construídos cultivados na remoção de matéria orgânica no tratamento de água residuária doméstica. Para isso, foram implantadas 18 unidades experimentais constituídos por cochos plásticos, em escala piloto, nas dimensões de $0,70 \mathrm{~m}$ de largura $\times 2,10 \mathrm{~m}$ de comprimento $\times 0,31 \mathrm{~m}$ de altura, e com declividade longitudinal de $0,05 \mathrm{~m} \mathrm{~m}-1$, preenchidos com meio suporte (brita \#1, D60=12 $\mathrm{mm}$, coeficiente de uniformidade - CU D60/D10 = 3,8 e volume de vazios inicial de 0,520m-3 m-3). Os sistemas foram submetidos a taxas de carregamento orgânico de 100 , 200 e $300 \mathrm{~kg}$ ha-1d-1 de DBO e diferentes tempos de detenção hidráulicos (1, 2 e 4 dias). As unidades foram cultivadas com forrageiras capim-elefante (Napier Pennisetum purpureum schum.), capim Mombaça (Megathyrsus maximus) e capim Tifton 85 (Cynodon spp.). Deste modo, as eficiências médias na remoção de sólidos totais, turbidez e DBO (Demanda bioquímica de oxigênio) ficaram entre 25 e $48 \%, 75$ a $96 \%$ e 59 a 76\%, respectivamente, havendo diferenças significativas entre leitos cultivados. Sendo o SAC10 responsável pelo melhor desempenho de remoção de sólidos e turbidez, e o SAC3 para remoção de DBO.
\end{abstract}

Palavras-chave: Tratamento; Água residuária; Ecológico; Baixo custo; Forragem.

\section{Performance of forage cultivated SACs for the removal of organic matter from domestic sewage}

\begin{abstract}
Among the alternatives for effluent treatment, aiming to minimize problems resulting from the lack of sanitation, the use of constructed wetlands has been highlighted, which, besides presenting low installation cost, being ecological, have easy to accept landscape effects for those who adhere to technique. Thus, this work aimed to evaluate the efficiency of cultivated flooded systems in the removal of organic matter in the treatment of domestic wastewater. For this, 18 experimental units consisting of plastic troughs were implemented, in pilot scale, in the dimensions of $0.70 \mathrm{~m}$ wide $\times 2.10 \mathrm{~m}$ long $\times 0.31 \mathrm{~m}$ high, and with longitudinal slope of $0.05 \mathrm{~m} \mathrm{~m}-1$, filled with half support (gravel \# 1, D60 $=12 \mathrm{~mm}$, uniformity coefficient - CU D60 / D10 = 3.8 and initial void volume $0.520 \mathrm{~m}-3 \mathrm{~m}-3$ ). The systems were submitted to organic loading rates of 100, 200 and $300 \mathrm{~kg}$ ha- $1 \mathrm{~d}-1$ BOD and different hydraulic detention times (1, 2 and 4 days). The units were cultivated with forage elephant grass (Napier - Pennisetum purpureum schum.), Mombasa grass (Megathyrsus maximus) and Tifton 85 grass (Cynodon spp.). Thus, the average efficiencies in total solids removal, turbidity and BOD (Biochemical Oxygen Demand) were between 25 and $48 \%, 75$ to $96 \%$ and 59 to $76 \%$, respectively, with significant differences between cultivated beds. Being SAC10 responsible for the best solids and turbidity removal performance, and SAC3 for BOD removal.
\end{abstract}

Keywords: Treatment; Waste water; Ecological; Low cost; Fodder.

Topic: Engenharia Agrícola

Reviewed anonymously in the process of blind peer
Received: 04/06/2019

Approved: 05/07/2019
Éllen Lemes Silva (iD)

Instituto Federal Goiano, Brasil

http://lattes.cnpq.br/2020996967548069

http://orcid.org/0000-0001-5649-5055

ellen cbba@hotmail.com

José Antônio Rodrigues de Souza (iD

Instituto Federal Goiano, Brasil

http://lattes.cnpq.br/1637300776838940

http://orcid.org/0000-0003-3024-9424

jose.antonio@ifgoiano.edu.br

Débora Astoni Moreira (it)

Instituto Federal Goiano, Brasil

http://lattes.cnpq.br/0892554192927049

http://orcid.org/0000-0002-8658-1269

debora.astoni@ifgoiano.edu.br

Matheus Rocha Mendes (1)
Instituto Federal Goiano, Brasil
http://lattes.cnpq.br/1430630633509461
$\begin{aligned} & \text { http://orcid.org/0000-0002-6308-7612 } \\ & \text { matheus engambiental@hotmail.com }\end{aligned}$
Diego César Veloso Rezende
Instituto Federal Goiano, Brasil
http://lattes.cnpq.br/2390623769547865
http://orcid.org/0000-0001-6176-0220
diegoformiga@yahoo.com.br
Walisson Marques Oliveira 10
Instituto Federal Goiano, Brasil
http://lattes.cnpq.br/7052217868475846
http://orcid.org/0000-0002-4615-8335
walissonmo.msn@hotmail.com

Matheus Rocha Mendes (iD) http://lattes.cnpq.br/1430630633509461 http://orcid.org/0000-0002-6308-7612

Diego César Veloso Rezende

Instituto Federal Goiano, Brasil

diegoformiga@yahoo.com.br

Walisson Marques Oliveira

Instituto Federal Goiano, Brasil http://orcid.

walissonmo.msn@hotmail.com

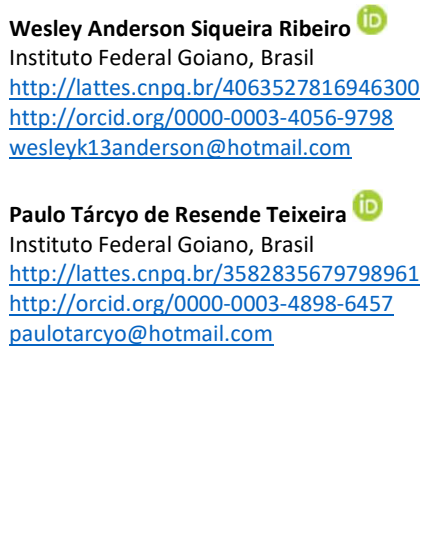

Wesley Anderson Siqueira Ribeiro Instituto Federal Goiano, Brasil http://orcid.org/0000-0003-4056-9798

Paulo Tárcyo de Resende Teixeira (ii) Instituto Federal Goiano, Brasil http://lattes.cnpq.br/3582835679798961 http://orcid.org/0000-0003-4898-6457 paulotarcyo@hotmail.com

DOI: 10.6008/CBPC2179-6858.2019.004.0004

Referencing this:

SILVA, É. L.; SOUZA, J. A. R.; MOREIRA, D. A.; MENDES, M. R.; REZENDE, D. C. V.; OLIVEIRA, W. M.; RIBEIRO, W. A. S.; TEIXEIRA, P. T. R.. Desempenho de SACs cultivados com forrageiras, quanto à remoção de matéria orgânica de esgoto doméstico. Revista lberoAmericana de Ciências Ambientais, v.10, n.4, p.36-44, 2019. DOI: http://doi.org/10.6008/CBPC2179-6858.2019.004.0004 


\section{INTRODUÇÃO}

Dentre os diversos recursos naturais disponíveis, a água tem se tornado o centro de discussões nesse início de século. Essencial para a manutenção da biodiversidade, é um dos principais elementos presentes na natureza e responsável pelo equilíbrio natural dos ecossistemas, tornando-se um recurso estratégico para a humanidade. Além de manter a vida no planeta, é fundamental na produção de alimentos, na agricultura, nas indústrias, na geração de energia, transporte etc.

Em virtude do seu uso indiscriminado, várias partes da Terra não dispõem deste recurso, seja pela sua escassez ou pela sua falta de qualidade. Nas cidades, os problemas de abastecimento estão diretamente relacionados ao crescimento urbano, ao desperdício e a urbanização não planejada. Já na zona rural, os recursos hídricos também são explorados de forma irregular, além de parte da vegetação em torno dos rios serem destruídas para a realização de atividades como agricultura e pecuária, existem os agrotóxicos e dejetos, que também acabam por poluir a água, impactando diretamente na sua qualidade.

Uma das alternativas para minimizar os problemas decorrentes da falta de saneamento nas áreas rurais é a implantação de tratamento de efluentes no local onde são produzidos, utilizando os sistemas naturais na interação. Nesse sentido, os sistemas de tratamento do tipo wetlands ou sistemas alagados construídos (SACs), têm sido apresentados como uma técnica ambiental economicamente viável para ser aplicada em comunidades rurais. Segundo Mendonça et al. (2015), este tipo de tratamento vem ganhando popularidade quando comparado com sistema convencional de tratamento, por demandar menor área para conseguir realizar o tratamento.

Trata-se de um processo natural, que utiliza os recursos disponíveis como a vegetação da própria região, não requer mão de obra especializada, apresenta baixo custo energético e é menos suscetível às variações nas taxas de aplicação de esgoto, exige baixa mecanização, energia e produtos químicos, aumenta o habitat para a vida animal e disponibiliza um elemento de estética paisagística (RAMOS et al., 2017), associando sua beleza, com o bom desempenho na depuração do esgoto, facilitando a aceitação da sociedade beneficiária.

Diversos trabalhos utilizando forrageiras vêm sendo aplicados no Brasil, como capins tifton 85 (Cynodon sp.) (SANTOS et al., 2016), grama esmeralda (MENDONÇA, 2017), capim elefante (Pennisetum sp.) (FORGIARINI et al., 2016), apresentando altas eficiências de remoção de nutrientes e poluentes de diversos tipos de águas residuárias.

Segundo FUNASA (2010), a relação de investimento em saneamento é de aproximadamente um (1) para quatro (4), ou seja, a cada $R \$ 1,00$ investido no setor, temos cerca de $R \$ 4,00$ economizados com saúde, o que mostra a grande importância da disposição adequada desses esgotos para a proteção da saúde pública. Diante disso, objetivou-se com este trabalho avaliar a eficiência de sistemas alagados construídos cultivados com gramíneas forrageiras no tratamento de água residuária doméstica quanto a remoção de matéria orgânica. 


\section{METODOLOGIA}

O trabalho foi conduzido no Instituto Federal Goiano - Campus Urutaí (IF Goiano), em Urutaí (GO), Brasil, localizado a $17^{\circ} 29^{\prime} 6^{\prime \prime} \mathrm{S}$ de latitude e $48^{\circ} 12^{\prime} 27^{\prime \prime} \mathrm{O}$ de longitude e $712 \mathrm{~m}$ de altitude. De acordo com a classificação de Köppen, o clima da região é do tipo Cwa, e se caracteriza como tropical úmido com inverno seco e verão chuvoso, com temperatura e precipitação médias, anuais, de $28^{\circ} \mathrm{C}$ e $2000 \mathrm{~mm}$ (SILVA et al., 2015).

Para condução dos ensaios experimentais, utilizou-se o esgoto doméstico proveniente das instalações do IF Goiano, o qual era captado em diversas dependências do Campus e conduzido até uma lagoa, sendo, posteriormente, bombeado e armazenado em caixa de 1000 litros para, então, serem distribuído por gravidade às unidades experimentais (sistemas alagados construídos - SAC, ou wetland). Na tabela 1, estão apresentados os resultados da caracterização do esgoto doméstico, realizado quinzenalmente, conforme metodologias descritas em APHA (2012).

Tabela 1: Caracterização física e química do esgoto doméstico utilizado nos ensaios experimentais.

\begin{tabular}{|c|c|}
\hline Parâmetros & Valores \\
\hline $\mathrm{pH}$ & $7,14 \pm 0,26$ \\
\hline Sódio total $\left(\mathrm{mg} \mathrm{L}^{-1}\right)$ & $1269 \pm 403$ \\
\hline Condutividade Elétrica (ds. $\left.\mathrm{cm}^{-1}\right)$ & $1,10 \pm 0,17$ \\
\hline Sólidos totais $\left(\mathrm{mg} \mathrm{L}^{-1}\right)$ & $684 \pm 62$ \\
\hline Turbidez $(\mathrm{UNT})$ & $345 \pm 98$ \\
\hline $\mathrm{DQO}\left(\mathrm{mg} \mathrm{O}_{2} \mathrm{~L}^{-1}\right)$ & $552 \pm 224$ \\
\hline $\mathrm{DBO}\left(\mathrm{mgO}_{2} \mathrm{~L}^{-1}\right)$ & $284 \pm 119$ \\
\hline Nitrogênio total $\left(\mathrm{mg} \mathrm{L}^{-1}\right)$ & $119 \pm 33$ \\
\hline Fósforo total $\left(\mathrm{mg} \mathrm{L}^{-1}\right)$ & $20 \pm 7$ \\
\hline Potássio total $\left(\mathrm{mg} \mathrm{L}^{-1}\right)$ & $233 \pm 17$ \\
\hline Cálcio total $\left(\mathrm{mg} \mathrm{L}^{-1}\right)$ & $64 \pm 14$ \\
\hline Magnésio Total $\left(\mathrm{mg} \mathrm{L}^{-1}\right)$ & $19 \pm 6$ \\
\hline Coliformes Totais (NMP 100 mL & $1.10^{6} \pm 7.10^{5}$ \\
\hline Coliformes Termotolerantes (NMP $\left.100 \mathrm{~mL}^{-1}\right)$ & $4.10^{5} \pm 3.10^{5}$ \\
\hline
\end{tabular}

Legenda: $\mathrm{pH}=$ potencial hidrogeniônico, $\mathrm{DBO}=$ demanda bioquímica de oxigênio, $\mathrm{DQO}=$ demanda bioquímica de oxigênio

A utilização destas espécies gramíneas em lugar das macrófitas aquáticas, ocorreu pelo fato de apresentarem boas características de produção e elevada capacidade de crescimento, além do grande potencial de uso como forrageira nas condições subtropicais e tropicais a se integrarem à paisagem local. Os wetlands utilizados, em escala piloto, foram constituídos por caixas plásticas (cochos em pvc de fundo plano), nas dimensões de $0,70 \mathrm{~m}$ de largura $\times 2,10 \mathrm{~m}$ de comprimento $\times 0,31 \mathrm{~m}$ de altura, assentado sobre o solo, em declividade longitudinal de $0,05 \mathrm{~m} \mathrm{~m}^{-1}$, preenchidos com meio suporte (brita \#1, D60=12 mm, coeficiente de uniformidade - CU D60/D10 =3,8 e volume de vazios inicial de $0,520 \mathrm{~m}^{-3} \mathrm{~m}^{-3}$ ) até a altura de $0,25 \mathrm{~m}$ em relação à base, e cultivados com uma espécie gramínea forrageira.

Cada SAC era dotado de sistema de drenagem, localizado na parte oposta à entrada do esgoto e ocupando toda largura da caixa, sendo confeccionado com tubo PVC de $32 \mathrm{~mm}$ de diâmetro e apresentando perfurações de aproximadamente $5 \mathrm{~mm}$ de diâmetro. Utilizou-se o escoamento subsuperficial horizontal $(0,25 \mathrm{~m}$ de lâmina de esgoto em escoamento no meio suporte de $0,31 \mathrm{~m}$ de altura), que foi garantindo por meio da instalação de tubulações reguladoras do nível do líquido instaladas na saída dos SACs. 
Os SACs foram saturados até uma altura de 0,20m com esgoto doméstico, permanecendo-se, assim, por 60 dias, objetivando-se a formação de biofilme para facilitar o pegamento das mudas dos capins. Neste período, propágulos vegetativos (rizomas + caule) com aproximadamente 0,30m de comprimento das gramíneas forrageiras capim Elefante Napier - Pennisetum purpureum schum., capim Mombaça Megathyrsus maximus e capim Tifton 85 - Cynodon spp., foram transplantados, uma espécie por SAC, em uma densidade de plantio média de 14 propágulos $\mathrm{m}^{-2}$.

Decorrido esse período, os ensaios experimentais foram iniciados, aplicando-se, diariamente, esgoto doméstico em diferentes cargas orgânicas aos SACs cultivadas com espécies de gramíneas, controlando-se a vazão por meio de válvulas de manobras (registro de gaveta) instaladas nas tubulações de condução do esgoto doméstico, posicionada à montante dos leitos de cada SAC. As medições para ajuste das vazões foram efetuadas pelo método direto, utilizando-se um recipiente graduado, sendo realizadas três vezes ao dia. 0 experimento foi conduzido no esquema fatorial $6 \times 3$ (6 cargas orgânicas e 3 espécies de gramíneas forrageiras) no delineamento inteiramente ao acaso, conforme tabela 2.

Tabela 2: Tratamentos avaliados na remoção da carga orgânica no esgoto doméstico.

\begin{tabular}{|c|c|c|c|}
\hline \multirow[b]{2}{*}{ Tratamentos } & \multicolumn{2}{|c|}{ Taxa de carga orgânica } & \multirow[b]{2}{*}{ Capins } \\
\hline & $\begin{array}{c}\text { Taxa de aplicação } \\
\left(\text { kg DBO }_{5} \text { dia }^{-1}\right)\end{array}$ & $\begin{array}{l}\mathrm{TDH} \\
\text { (dias) }\end{array}$ & \\
\hline $\mathrm{SAC}_{1}$ & 100 & 5,6 & Elefante \\
\hline $\mathrm{SAC}_{2}$ & 100 & 5,6 & Mombaça \\
\hline $\mathrm{SAC}_{3}$ & 100 & 5,6 & Tifton \\
\hline $\mathrm{SAC}_{4}$ & 200 & 2,8 & Tifton \\
\hline $\mathrm{SAC}_{5}$ & 200 & 2,8 & Elefante \\
\hline $\mathrm{SAC}_{6}$ & 200 & 2,8 & Mombaça \\
\hline $\mathrm{SAC}_{7}$ & 300 & 1,9 & Mombaça \\
\hline $\mathrm{SAC}_{8}$ & 300 & 1,9 & Tifton \\
\hline $\mathrm{SAC}_{9}$ & 300 & 1,9 & Elefante \\
\hline $\mathrm{SAC}_{10}$ & 140,63 & 4,0 & Elefante \\
\hline $\mathrm{SAC}_{11}$ & 140,63 & 4,0 & Mombaça \\
\hline $\mathrm{SAC}_{12}$ & 140,63 & 4,0 & Tifton \\
\hline $\mathrm{SAC}_{13}$ & 281,25 & 2,0 & Tifton \\
\hline $\mathrm{SAC}_{14}$ & 281,25 & 2,0 & Elefante \\
\hline $\mathrm{SAC}_{15}$ & 281,25 & 2,0 & Mombaça \\
\hline $\mathrm{SAC}_{16}$ & 562,5 & 1,0 & Tifton \\
\hline $\mathrm{SAC}_{17}$ & 562,5 & 1,0 & Mombaça \\
\hline $\mathrm{SAC}_{18}$ & 562,5 & 1,0 & Elefante \\
\hline
\end{tabular}

Legenda: SAC- sistema alagado construído, TDH- Tempo de detenção hidráulico

Foram realizadas amostragens quinzenais do esgoto doméstico e dos efluentes dos SACs, sendo o sistema de tratamento monitorado por 110 dias. As amostras foram conduzidas ao Laboratório de Pesquisa e Análises Químicas do IF Goiano, para determinação de turbidez; condutividade elétrica (CE); potencial hidrogeniônico $(\mathrm{pH})$; nitrogênio total, potássio, cálcio, magnésio, sódio, fósforo total, demanda bioquímica de oxigênio (DBO), demanda química de oxigênio (DQO), sólidos totais, coliformes totais e termotolerantes, conforme metodologias descritas em APHA (2012).

\section{RESULTADOS E DISCUSSÃO}

Na Figura 1, estão apresentadas as eficiências de remoção de matéria orgânica (ST, Turbidez e DBO) durante o período de monitoramento do sistema. Verifica-se que a eficiência de remoção de sólidos totais (ST) referentes aos primeiros 45 dias avaliados foram menores que os demais, isso pode estar relacionado à 
fase inicial de desenvolvimento vegetativo, principalmente crescimento radicular e adaptação das plantas ao sistema. Uma vez que, a presença dos rizomas e raízes proporcionam redução de velocidade do escoamento e distribuição do efluente no interior dos SACs. Assim como observado por Mendonça (2015) e Oliveira et al. (2018), os quais justificam que o maior tempo de contato entre a água e as raízes das plantas, propiciam melhores condições para a sedimentação de sólidos em suspensão e degradação de partículas orgânicas pelos microrganismos.

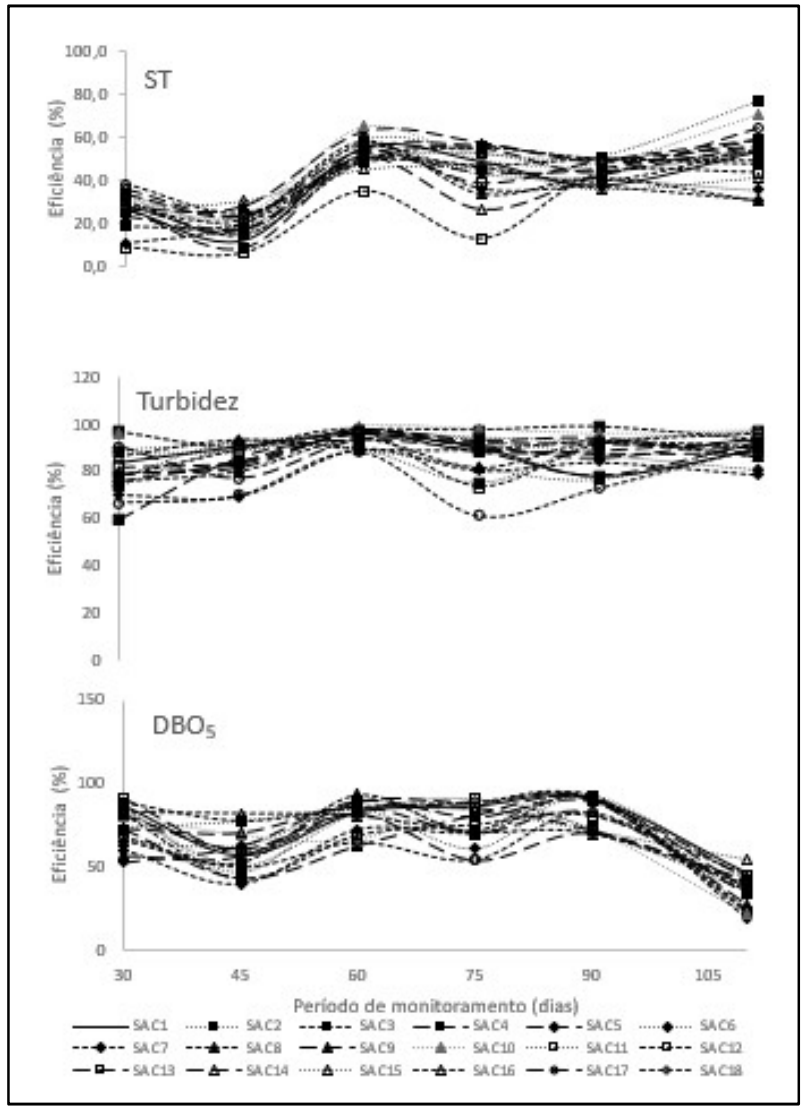

Figura 1: Eficiências de remoção de $\mathrm{ST}$, turbidez e $\mathrm{DBO}_{5}$, nos $\mathrm{SACs}$, durante o monitoramento dos sistemas.

As oscilações na remoção de sólidos podem estar relacionadas as variações de concentrações do afluente verificado ao longo do monitoramento. Verifica-se ainda, que no final do monitoramento, houve tendência de maior remoção para sólidos, o que pode estar correlacionado com a poda dos capins, bem como desenvolvimento de novas raízes aumentando a eficiência dos processos de filtração, absorção e decantação.

Segundo Mendonça (2015), Matos (2015), Lourenço (2017), os SACs apresentam-se eficientes na remoção de sólidos suspensos totais, sendo os processos responsáveis por isso: sedimentação nos interstícios, adesão aos grânulos do material suporte (devido a ação da força de van der Waals) e filtração, os quais apresentam essas características devido ao uso de maiores tempos de detenção hidráulicos, presença de meio suporte e vegetais.

Referente ao parâmetro turbidez, verifica-se tendência de estabilização na eficiência de remoção ao longo do monitoramento do sistema (figura 1). Uma vez que este parâmetro está correlacionado aos sólidos, pode-se dizer que os sistemas plantados proporcionaram altas eficiências, corroborando com Almeida 
(2016), o qual pôde verificar que a remoção de sólidos interfere na remoção de turbidez, sendo estes decorrentes do processo de filtração pelo substrato e sistema radicular.

Observa-se, ainda na figura 1, que os SACs apresentaram bastante estáveis até 90 dias após transplantio; posterior a este período, nota-se um decréscimo na remoção de DBO. Isso pode estar relacionado com a poda/corte das forrageiras nos SACs, assim como verificado por Lourenço (2017), o qual pôde constatar a baixa eficiência na remoção de DBO, nos períodos pós-poda atribuindo esse fato aos restos vegetais sob o leito e no seu interior (sistema radicular em decomposição).

Um dos fatores que provavelmente contribuíram para a rápida estabilização do sistema, foi o desenvolvimento do biofilme no meio suporte, gerado via aplicação de esgoto doméstico em todos os SACs, durante 60 dias antes do início dos ensaios experimentais. Como notado por Saraiva (2016), que após aplicar o efluente por 45 dias, possibilitou uma maior estabilização na remoção de poluentes, atribuindo a um possível grau de maturação do biofilme ser maior. Segundo Mendonça (2015), a diminuição da vazão aplicada de esgotos e a elevação do TDH influenciam positivamente em relação às remoções dos parâmetros físicoquímicos e microbiológicos.

Na tabela 3, estão apresentadas as eficiências de remoção da matéria orgânica, suas concentrações médias e o desvio-padrão representados por ST, Turbidez e DBO, afluente e efluente dos SACs. Nota-se que as eficiências de remoção de ST, variaram de 25 a 48\%, os quais apresentaram diferença estatisticamente significativa para os tratamentos. Dentre os SACs, o tratamento que apresentou maior eficiência de remoção tanto para sólidos totais quanto para turbidez, foi o $\mathrm{SAC}_{10}$ com tempo de detenção hidráulico de 4 dias e taxa de carga orgânica de 140,6kg.ha-1 $\mathrm{dia}^{-1}$ de DBO.

Isso pode estar relacionado a forrageira utilizada (capim-elefante), a qual apresentou características de sistema radicular com maior densidade de suas ramificações no início da unidade experimental (cocho), com a altura de aproximadamente de $17 \mathrm{~cm}$ e crescimento vertical (raiz), e também ao tempo de detenção, os quais em conjunto contribuem para redução da velocidade de escoamento do esgoto no interior do SAC, e processos físicos de filtração e sedimentação das partículas, como encontrado por Correa (2018) onde verificou a maior remoção nos SACs com menores concentrações aplicadas; já Lourenço (2017) e Ansari et al. (2016), atribuem essa maior remoção causada devido a retenção de partículas no meio poroso utilizado.

Tabela 3: Valores médios e desvio-padrão dos afluentes e efluentes dos SACs e eficiências médias (\%) de remoção e desvio-padrão de sólidos totais (ST), Turbidez e demanda bioquímica de oxigênio (DBO) nos SACs obtidos durante a condução do experimento

\begin{tabular}{|c|c|c|c|c|c|c|}
\hline \multirow[b]{2}{*}{ TRAT } & \multicolumn{2}{|c|}{ ST } & \multicolumn{2}{|c|}{ Turbidez } & \multicolumn{2}{|c|}{ DBO } \\
\hline & $\left(\mathrm{mg} \mathrm{L}^{-1}\right)$ & Rem. (\%) & $\left(\mathrm{mg} \mathrm{L}^{-1}\right)$ & Rem. (\%) & $\left(\mathrm{mg} \mathrm{L}^{-1}\right)$ & Rem. (\%) \\
\hline Afluente & $684 \pm 62$ & & $300 \pm 98$ & & $284 \pm 119$ & \\
\hline SAC1 & $415 \pm 113$ & $39 \pm 17 K$ & $38 \pm 26$ & $88 \pm 61$ & $76 \pm 76$ & $75 \pm 18 B$ \\
\hline SAC2 & $360 \pm 149$ & $48 \pm 22 B$ & $39 \pm 32$ & $86 \pm 9 L$ & $85 \pm 83$ & $71 \pm 22 D$ \\
\hline SAC3 & $420 \pm 105$ & $39 \pm 15 \mathrm{~L}$ & $12 \pm 8$ & $96 \pm 3 B$ & $81 \pm 118$ & $76 \pm 26 \mathrm{~A}$ \\
\hline SAC4 & $398 \pm 136$ & $42 \pm 20 \mathrm{H}$ & $47 \pm 27$ & $83 \pm 13 N$ & $101 \pm 85$ & $64 \pm 210$ \\
\hline SAC5 & $415 \pm 114$ & $40 \pm 16 \mathrm{~J}$ & $34 \pm 13$ & $88 \pm 61$ & $90 \pm 76$ & $69 \pm 19 \mathrm{H}$ \\
\hline SAC6 & $429 \pm 89$ & $37 \pm 130$ & $33 \pm 23$ & $89 \pm 6 F$ & $107 \pm 110$ & $64 \pm 24 N$ \\
\hline SAC7 & $466 \pm 102$ & $32 \pm 16 Q$ & $61 \pm 20$ & $79 \pm 80$ & $117 \pm 113$ & $59 \pm 26 Q$ \\
\hline SAC8 & $440 \pm 90$ & $36 \pm 13 P$ & $43 \pm 19$ & $85 \pm 7 \mathrm{M}$ & $102 \pm 109$ & $66 \pm 26 \mathrm{~K}$ \\
\hline SAC9 & $368 \pm 99$ & $46 \pm 15 C$ & $31 \pm 15$ & $89 \pm 7 G$ & $96 \pm 105$ & $68 \pm 261$ \\
\hline SAC10 & $358 \pm 128$ & $48 \pm 19 A$ & $11 \pm 7$ & $96 \pm 3 A$ & $107 \pm 113$ & $66 \pm 24 \mathrm{~J}$ \\
\hline SAC11 & $395 \pm 92$ & $42 \pm 13 F$ & $25 \pm 13$ & $92 \pm 3 C$ & $96 \pm 93$ & $70 \pm 22 F$ \\
\hline
\end{tabular}




\begin{tabular}{|c|c|c|c|c|c|c|}
\hline SAC12 & $519 \pm 142$ & $25 \pm 17 \mathrm{R}$ & $38 \pm 36$ & $87 \pm 10 \mathrm{~J}$ & $100 \pm 70$ & $65 \pm 17 \mathrm{~L}$ \\
\hline SAC13 & $391 \pm 102$ & $43 \pm \mathrm{E} 13$ & $30 \pm 11$ & $89 \pm 6 \mathrm{E}$ & $89 \pm 85$ \\
\hline SAC14 & $434 \pm 117$ & $37 \pm 15 \mathrm{~N}$ & $37 \pm 16$ & $87 \pm 7 \mathrm{~K}$ & $95 \pm 91$ \\
\hline SAC15 & $423 \pm 45$ & $38 \pm 8 \mathrm{M}$ & $46 \pm 28$ & $85 \pm 7 \mathrm{M}$ & $81 \pm 66$ & $69 \pm 19 \mathrm{G}$ \\
\hline SAC16 & $401 \pm 109$ & $41 \pm 16 \mathrm{I}$ & $29 \pm 10$ & $90 \pm 3 \mathrm{D}$ & $96 \pm 87$ & $75 \pm 12 \mathrm{~B}$ \\
\hline SAC17 & $372 \pm 99$ & $46 \pm 15 \mathrm{D}$ & $33 \pm 16$ & $88 \pm 7 \mathrm{H}$ & $109 \pm 79$ & $65 \pm 16 \mathrm{M}$ \\
\hline SAC18 & $397 \pm 72$ & $42 \pm 10 \mathrm{G}$ & $75 \pm 47$ & $75 \pm 12 \mathrm{P}$ & $116 \pm 79$ & $59 \pm 17 \mathrm{P}$ \\
\hline
\end{tabular}

Legenda: TRAT- tratamentos, Rem. - Porcentagem de remoção das variáveis tendo como base o balanço de massa realizado. Médias seguidas de mesma letra na coluna não diferem significativamente entre si, a 5\% de probabilidade, pelo teste de Tukey. ST- Sólidos totais; DBO- demanda bioquímica de oxigênio.

Foi possível observar também diferenças estatísticas dos tratamentos em relação aos tipos de capins utilizados, uma vez que para a mesma taxa de aplicação os sistemas diferiram. O SAC 10 cultivado com capim elefante houve maior remoção (48\%), e o $\mathrm{SAC}_{12}$ (capim-tifton) com a mesma taxa aplicada, apresentou o menor índice de remoção de $25 \%$. Isto pode ser explicado uma vez que, as características físicas das raízes principais do capim-tifton 85 apresentarem crescimento vertical e baixa densidade de ramificações, podendo haver formação de caminhos preferenciais que facilitem a passagem das partículas e maior velocidade de escoamento no interior do leito. Tal fato foi verificado, também, por Fia et al. (2016).

Correa (2018), em seu trabalho verificou tendência de maior remoção em SACs vegetados, o qual justificou como fator positivo no tratamento de água residuária de suinocultura a forrageira utilizada(capimJiggs). No entanto, Fia (2009) relata menor eficiência de remoção observada em SACs vegetados, uma vez que na rizosfera, há possibilidade de gerar caminhos que facilitam a passagem de partículas, levando à menor eficiência, além disso, segundo Lourenço (2017) a baixa eficiência pode ocorrer devido restos vegetais sob o leito e no seu interior nos períodos pós-poda.

Nota-se durante a fase de aplicação de água residuária doméstica, que nenhum dos capins apresentaram sinais de senescência e intoxicação mesmo com a maior taxa de DBO aplicada (562,5kg.ha $\left.{ }^{1} \mathrm{dia}^{-1}\right)$. No entanto, Silva (2014) verificou tal distúrbio após um período de intermitência na alimentação do sistema de aplicação de água residuária doméstica. As médias de concentrações do afluente ao longo do período de monitoramento foi de $284 \mathrm{mg}^{\mathrm{L}^{-1}}$, semelhante ao encontrado por Correa (2018) com tratamento de água residuária de suinocultura o qual foi de $282 \mathrm{mg} \cdot \mathrm{L}^{-1}$, assim essa carga não pode ser considerada como carga orgânica tão elevada, quando comparada à outros estudos (Matos et al., 2012; Santos et al., 2016).

Em relação a DBO, nota-se que houve diferença significativa para eficiências de remoção (Tabela 3), entre as cargas orgânicas aplicadas nos dezoitos SACs avaliados. Observou-se também, que a menor taxa de carga orgânica aplicada (100 kg ha $\left.{ }^{-1} \mathrm{dia}^{-1} \mathrm{DBO}\right)$, apresentou maior eficiência de remoção e menor concentração de $\mathrm{DBO}$. Com eficiência de $76 \%$ no $\mathrm{SAC}_{3}$, já no $\mathrm{SAC}_{18}$ com a aplicação da maior carga orgânica e menor tempo de detenção hidráulico houve menor eficiência média de remoção (59\%). Isso pode ser atribuído ao fato de o maior tempo de retenção do esgoto no interior do SAC, ser ideal para sedimentação do material orgânico, promovendo a proliferação e desenvolvimento das bactérias que degradam sólidos coloidais e orgânicos solúveis, inferindo em maior eficiência na remoção.

Assim como Correa (2018) pôde averiguar em seu estudo, onde a carga aplicada de $100 \mathrm{~kg} \cdot \mathrm{ha}^{-1} \cdot{ }^{-1 i a-}{ }^{1}$, com presença de vegetação apresentou menores concentrações de DBO. Por outro lado, Fia (2009), pôde constatar que o maior TDH aplicado aos SACs, faz com que haja retenção de matéria orgânica que já passou 
por processo de degradação no interior do sistema, apresentando maior quantidade de compostos recalcitrantes de difícil degradação, responsável por reduzir a eficiência.

Comparando os resultados obtidos com alguns trabalhos presentes na literatura, verifica-se maior eficiência de remoção de DBO neste estudo. Forgiarini et al. (2016), avaliando remoção de matéria orgânica biodegradável, Lourenço (2017) e Baskar et al. (2014) no tratamento de esgoto doméstico; com eficiências de remoção de 53\%; 64\%, e 66,2\%, respectivamente.

Por outro lado, autores como Nagamati et al. (2018), com tratamento de efluente doméstico, apresentaram eficiência média de 79\% e Saraiva (2016) no tratamento de efluente de tanque de resfriamento de leite conseguiu máxima eficiência de 97\% e mínima de 55\%, superior ao encontrado neste trabalho. Assim, as eficiências na degradação e consequentemente, na remoção da matéria orgânica estão atreladas a vários fatores: TDH, tipo de material suporte utilizado e granulometria, características do afluente, taxas orgânicas aplicadas, condições climáticas, espécies vegetais, assim estes fatores devem ser levados em consideração ao se comparar diferentes dados de literatura (SARAIVA, 2016).

Segundo Mendonça (2015) e Poças (2015), o fator tempo é responsável principalmente, por ativar e acelerar os processos microbianos heterotróficos, ou seja, quanto maior o tempo de operação do SAC, maior a eficiência de remoção de matéria orgânica. Entretanto, Matos et al. (2012) verificou que o aumento nas eficiências de remoção de matéria orgânica, em sistemas de tratamento, pode ocorrer com aumento na carga orgânica aplicada.

Dentre os fatores responsáveis pela maior eficiência de remoção de matéria orgânica em sistemas de tratamento, destacou-se o tempo de detenção hidráulico de 5,6 dias e a forrageira capim tifton 85 . Sendo este, próximo ao encontrado por Baskar et al. (2014), com TDH de 6dias no tratamento de água residuária doméstica. E superior ao verificado por Ramos et al. (2016) e Queluz (2016), TDH=3,2 dias, no tratamento de água residuária de suinocultura e água residuária doméstica, respectivamente. Contudo, há exceção do $\mathrm{SAC}_{18}$, os resultados obtidos atenderam a resolução CONAMA n.430/2011 (CONAMA, 2011) a qual preconiza uma eficiência de remoção mínima de $60 \%$ para DBO, assim o uso de SACs podem ser considerados excelente alternativa para tratamento de efluente doméstico.

\section{CONCLUSÕES}

Conclui-se que para as condições experimentais as espécies de forrageiras utilizadas se mostraram adequadas à utilização em sistemas de tratamento do tipo wetlands, atendendo a legislação vigente. Dentre os sistemas avaliados, o com maior eficiência de remoção tanto para sólidos totais e para turbidez, foi o $\mathrm{SAC}_{10}$ com tempo de detenção hidráulico de 4 dias e taxa de carga orgânica de $140,6 \mathrm{~kg}_{\text {. }} \mathrm{ha}^{-1} \mathrm{dia}^{-1} \mathrm{de} \mathrm{DBO}$ e cultivado com capim elefante. Em relação ao parâmetro $\mathrm{DBO}$ o $\mathrm{SAC}_{3}$ com menor taxa de carga orgânica avaliada (100kg ha-1 $\left.\mathrm{dia}^{-1} \mathrm{DBO}\right)$, apresentou maior eficiência de remoção e menor concentração de DBO.

\section{REFERÊNCIAS}


ANSARI, A. A.; GILL, R.; GILL, S. S.; LANZA, G. R.. Phytoremediation. Management of Environmental Contaminants, v.4, 2016.

APHA. American Public Health Association. Standard Methods for the Examination of Water and Wastewater. 22 ed. New York: APHA, 2012.

BASKAR, G.; DEEPTHA, V. T.; ANNADURAI, R.. Comparison of treatment performance between constructed wetlands with different plants. International Journal of Research in Engineering and Technology, v.3, p.210-214. DOI: http://doi.org/10.15623/ijret.2014.0304037

CONAMA. Conselho Nacional do Meio Ambiente. Resolução n.430 de 13 de maio de 2011. Brasília: DOU, 2011.

CORREA, J. M.. Avaliação de sistemas alagados construídos no tratamento de água residuária de suinocultura. Dissertação (Mestrado) - Universidade Federal do Espírito Santo, Alegre, 2018.

FIA, F. R. L.. Modelos de remoção de matéria orgânica e nutrientes de águas residuárias da suinocultura em sistemas alagados construídos. Tese (Doutorado) Universidade Federal de Viçosa, Viçosa, 2009.

FIA, F. R. L.; MATOS, A. T.; FIA, R.; BORGES, A. C.; CECON, P. R.. Efeito da vegetação em sistemas alagados construídos para tratar águas residuárias da suinocultura. Engenharia Sanitária e Ambiental, v.22, n.2, p.303-311, 2016. DOI: http://doi.org/10.1590/s1413-41522016123972

FORGIARINI, F. R.; RIZZI, E. S.. Eficiência de diferentes macrófitas na remoção da matéria Orgânica biodegradável em 'wetland' construído de fluxo Vertical em clima subtropical. Ciência \& Engenharia, v.25, n.2, p.79-86, 2016.

FUNASA. Fundação Nacional de Saúde. Manual de saneamento. Brasília: FUNASA, 2010.

LOURENÇO, L. S.. Remoção de matéria orgânica e nutrientes de esgoto doméstico por wetland na estação de tratamento aparecida - Campos Novos. Dissertação (Mestrado) - Universidade do Estado de Santa Catarina, Lages, 2017.

MATOS, A. T.. Manual de análise de resíduos sólidos e águas residuárias. Viçosa: EDUFV, 2015.

MATOS, A. T.; ABRAHÃO, S. S.; MONACO, P. A. V.. Eficiência de sistemas alagados construídos na remoção de poluentes de águas residuárias da indústria de laticínios. Engenharia Agrícola, v.32, n.6, p.1144-1155, 2012. DOI: http://doi.org/10.1590/S0100-69162012000600016

MENDONÇA, A. A. J.. Avaliação de um sistema descentralizado de tratamento de esgotos domésticos em escala real composto por tanque séptico e wetland construída híbrida. Dissertação (Mestrado) - Universidade de São Paulo, São Paulo, 2015.

MENDONÇA, H. V.; RIBEIRO, C. B. M.; NOGUEIRA, K. C. C.. Remoção de matéria orgânica e nutrientes de águas residuais de laticínios em sistemas alagados construídos. Revista de Ciências Agrárias, v.40, n.1, p.12-22, 2017. DOI: http://doi.org/10.19084/RCA15154

NAGAMATI, F. L.; SANTOS, J. J. F.; MENDES, T. A.. Execução de wetland construído para tratamento de efluente doméstico em propriedade rural. Gestão \& Sustentabilidade Ambiental, Florianópolis, v.7, n.1, p.418-446, 2018. DOI: http://doi.org/10.19177/rgsa.v7e12018418-446

OLIVEIRA, L. P.; SOUZA, M. B.; SOARES, A. F. S.; ANDRADE, I. C. M.. Avaliação da eficiência de Wetlands na remoção de matéria orgânica de esgoto sanitário. NBC, v.8, n.15, 2018.

POÇAS, C. D.. Utilização da tecnologia de wetlands para tratamento terciário: controle de nutrientes. Dissertação (Mestrado) - Universidade de São Paulo, São Paulo, 2015.

QUELUZ, J. G. T.. Eficiência de alagados construídos para o tratamento de águas residuárias com baixas cargas orgânicas. Tese (Doutorado) - Universidade do Estado de São Paulo, Botucatu, 2016.

RAMOS, N. F. S.; BORGES, A. C.; GONÇALVES, G. C.; MATOS, A. T.. Tratamento de águas residuárias de suinocultura em sistemas alagados construídos, com Chrysopogon zizanioides e Polygonum punctatum cultivadas em leito de argila expandida. Engenharia Sanitária e Ambiental, v.22, n.1, p.123-132, 2017. DOI: http://doi.org/10.1590/s1413$\underline{4152201687067}$

SANTOS, B. S.; COSTA, P. F.; EYNG, E.; CÂMARA, C. D.. Avaliação da Eficiência de um Sistema de Tratamento por Wetland Construído Aplicado ao Efluente de um Frigorífico de Suínos. Semina: Ciências Exatas e Tecnológicas, v.37, n.2, p.13-22, 2016. DOI: http://doi.org/10.5433/1679$0375.2016 \mathrm{v} 37 \mathrm{n} 2 \mathrm{p} 13$

SANTOS, V. K. S.; COLARES, A. P. F.; MARTINS, R. N.; SILVA, V. V.; SOUZA, M. A. R.; RIBEIRO, D. P.. Eficiência de sistemas alagados construídos na remoção de poluentes de águas residuárias da suinocultura. In: ENCONTRO LATINO AMERICANO DE INICIAÇÃO CIENTÍFICA, 20. Anais. Urbanova: Universidade do Vale do Paraíba, 2016.

SARAIVA, C. B.. Tratamento de água residuária de tanque de resfriamento de leite utilizando sistema alagado construído com diferentes meios suporte e disposição das espécies vegetais cultivadas. Tese (Doutorado) Universidade Federal de Viçosa, Viçosa, 2016.

SILVA, L. C. L.. Sistema biológico combinado (wetlands+ biofiltros) para o tratamento de efluente doméstico bruto. Monografia (Graduação) - Universidade Católica de Brasília, Brasília, 2014.

A CBPC - Companhia Brasileira de Produção Científica (CNPJ: 11.221.422/0001-03) detém os direitos materiais desta publicação. Os direitos referem-se à publicação do trabalho em qualquer parte do mundo, incluindo os direitos às renovações, expansões e disseminações da contribuição, bem como outros direitos subsidiários. Todos os trabalhos publicados eletronicamente poderão posteriormente ser publicados em coletâneas impressas sob coordenação da Sustenere Publishing, da Companhia Brasileira de Produção Científica e seus parceiros autorizados. Os (as) autores (as) preservam os direitos autorais, mas não têm permissão para a publicação da contribuição em outro meio, impresso ou digital, em português ou em tradução. 